\title{
4 Nach Gallipoli: der Erste Weltkrieg im Mittleren Osten
}

Mit dem Sieg des osmanischen Heeres unter deutscher Führung bei Gallipoli war der Erste Weltkrieg im Mittleren Osten nicht beendet, denn selbst alliierten Befehlshabern sowie den politisch Verantwortlichen in London und Paris war klar geworden, dass es einen leichten Sieg auch auf diesem Kriegsschauplatz nicht geben würde. Ebenso setzten die deutschen Offiziere und Mannschaften, bis Oktober 1918 immerhin mehr als 20.000 von ihnen, ihre Bemühungen fort, den osmanischen Verbündeten nach Kräften zu unterstützen. ${ }^{1}$ Während der Oberbefehl der Streitkräfte nominell bei Sultan Mehmed V. Reşat (1844-1918) lag, war der eigentliche Entscheidungsträger auf Seiten der Osmanen immer noch Enver Pascha. Dieser ernannte Bronsart von Schellendorf zu Kriegsbeginn zum Chef des Generalstabes des Heeres. ${ }^{2}$ Das schränkte Liman von Sanders Einfluss ein, ${ }^{3}$ obwohl letzterer sich als Oberbefehlshaber um die Verteidigung der Dardanellen und der Gallipoli-Halbinsel zu kümmern hatte. Im Zuge dieser Kampagne hatten die osmanischen Soldaten bewiesen, dass sie durchaus „diszipliniert, standhaft und zäh“4 waren und so die Wahrnehmung vieler deutscher Offiziere bestärkt, dass es nur ausreichender Vorbereitungszeit sowie ordentlicher Führung bedurfte, um ihr volles militärisches Potential abzurufen.

Generell hatte die Arbeit der deutschen Militärmission dazu beigetragen, ein modernes Heer aufzustellen, dessen Chancen zu Beginn der Kriegshandlungen 1914 nicht die schlechtesten waren. Während der Zustand des Heeres auf deutsche Modernisierungsmaßnahmen zurückging, waren die Aufmarschpläne von Friedrich Bronsart von Schellendorf konzipiert worden, wobei er sich in erster Linie auf die Mobilmachung, den Schutz der westlichen Grenzen sowie die Konzentration von Truppen in geostrategisch wichtigen Regionen konzentriert hatte. ${ }^{5}$

In letzterem Fall teilten Enver und Liman von Sanders die Einschätzung, dass die Verteidigung der Dardanellen und der Gallipoli-Halbinsel, denen Bronsart von Schellendorf „überragende strategische Bedeutung “ ${ }^{\text {“6 }}$ beigemessen hatte, oberste

1 Krethlow, Bagdad, S. 8.

2 Ebd., S. 16.

3 Dessen ungeachtet kam es zwischen Enver Pascha und Liman von Sanders auch in den folgenden Jahren immer wieder zu Streitigkeiten um die jeweiligen Kompetenzen. Vgl. dazu Enver Pascha an Liman von Sanders, 28. Januar 1916, BArch MArch RH61/1088.

4 Krethlow, Bagdad, S. 17.

5 Ebd., S. 19.

6 Ebd.

Ә OpenAccess. ( 2020 Frank Jacob, publiziert von De Gruyter. (cc) BY-NC-ND Dieses Werk ist lizenziert unter der Creative Commons Attribution-NonCommercial-NoDerivatives 4.0 License.

https://doi.org/10.1515/9783110694772-004 
Priorität besäßen. ${ }^{7}$ Kaum waren diese erfolgreich gegen den britischen Invasionsversuch verteidigt worden, brachen die Konflikte allerdings wieder auf, gerade weil Enver, im Gegensatz zu den Plänen des Chefs des Generalstabes und auch entgegen der Ideen Liman von Sanders keinen defensiv ausgerichteten, sondern einen aggressiven Krieg zu führen gedachte. Im Zuge dessen sollte der osmanische Einfluss durch Angriffe im Kaukasus und gegen den Suezkanal ausgeweitet werden. Im Folgenden sollen die weiteren Operationen des Weltkrieges im Kaukasus und im Mittleren Osten, d.h. in Palästina und Ägypten sowie in Mesopotamien bzw. dem Irak kurz zusammengefasst werden. ${ }^{8}$ Im Vergleich dazu wird schnell deutlich, warum Gallipoli aus deutscher und osmanischer Sicht eine Sonderstellung innerhalb des Kriegsgeschehens an diesen Fronten einnahm.

\subsection{Kaukasus}

Liman von Sanders hatte die allgemeine Lage nach der erfolgreichen GallipoliKampagne als durchaus solide eingeschätzt. Später würde er die Aktionen Enver Paschas im Kaukasus für die schließlich so schlechte Lage der osmanischen Armee gegen Kriegsende verantwortlich machen. Mühlmann schrieb mit Blick auf die Darstellung des Reichsarchivs zum Weltkrieg im Mittleren Osten 1929: „So schlimm wie die Lage der Türkei [...] dargestellt ist, war sie damals nicht. Sie wurde erheblich erst verschlechtert, als Enver die gute III. Armee im Kaukasus (ca.90.000 Mann) fast gänzlich zu Grunde richtete. “9 Ungeachtet der Differenzen, die Liman von Sanders mit dem Kriegsminister des Osmanischen Reiches pflegte, war diese Einschätzung durchaus richtig. Die militärischen Operationen im Kaukasus sollten das osmanische Heer maßgeblich schwächen, zumal zwei Drittel aller Verluste des Heeres zwischen November 1915 und Februar 1917 in dieser Region verzeichnet wurden. Dies hatte zudem zur Folge, dass die Kontingente der Armee bis 1917 derartig geschwächt worden waren, dass sie den anlaufenden britischen Kampagnen in Palästina und Mesopotamien nur noch wenig entgegenzusetzen hatte.

Die Grundvoraussetzungen im Kaukasus waren dabei eigentlich durchaus passabel, bedenkt man, dass die russische Kaukasusarmee erschöpft war, denn die Hälfte der verfügbaren Truppen hatten nach dem Erfolg des deutschen Heeres

7 Militär-Attaché der Kaiserlich Deutschen Botschaft, Major von Laffert, 160 Pers[onen], Pera,

2. März 1914, BArch MArch RH61/1088.

8 Die Schilderung folgt dabei, sofern nicht anders angegeben, Ulrichsen, First World War, S. 53-73 (Kaukasus), S. 97-118 (Palästina und Ägypten) und S. 119-146 (Mesopotamien). 9 Liman von Sanders an Carl Mühlmann, München, 3. Juli 1929, BArch MArch RH61/1802. 
bei Tannenberg und an den Masurischen Seen 1914 in Richtung Westen verlagert werden müssen. Sowohl das russische als auch das osmanische Oberkommando mussten folglich mit mehreren Fronten und deren spezifischen Notwendigkeiten planen. Beide Seiten erhofften sich aber einen Sieg in einem Waffengang gegen den scheinbar bezwingbaren Gegner.

Der osmanische Vormarsch auf Sarıkamış begann am 22. Dezember 1914 und startete die erste Offensive des Heeres gegen den russischen Feind. Der Jahreszeit entsprechend wurden die Truppenbewegungen in dieser Region durch schwere Schneestürme und generell sehr niedrige Temperaturen, an die die meisten Soldaten nicht gewöhnt waren, erschwert. Die Märsche durch bergiges Terrain ließen die Moral der Truppen sinken, zumal die Logistik des Heeres ebenfalls vor eine schwierige Aufgabe gestellt wurde und die ausreichende Versorgung mit Proviant und anderen notwendigen Ausrüstungsgegenständen kaum zu bewerkstelligen war. Die Idee Enver Paschas war es, die russischen Kontingente einzuschließen und mit einem Durchstoß bei Sarıkamış, der die Kommunikationslinie des Feindes unterbrochen hätte, zur Aufgabe zu zwingen. Dieser Plan war möglicherweise vom deutschen Sieg bei Tannenberg inspiriert worden, die notwendige Mobilität für eine solche Operation war allerdings nicht vorhanden. Der Plan mag auf dem Papier und unter Vorstellung eines schnellen und reibungslosen osmanischen Vormarsches durchaus überzeugt haben. In den realen Winterbedingungen des Kaukasus war jedoch nicht an ein schnelles Vorrücken, geschweige denn erfolgreiches Einkreisen des russischen Feindes zu denken. Ohne Rücksicht auf etwaige Zweifel an den Erfolgsaussichten rückten am 22. Dezember 1914 schließlich 118.000 osmanische Soldaten der Dritten Armee gegen 65.000 russische vor. Die numerische Überlegenheit darf hier nicht überbewertet werden, waren Enver Paschas Truppen doch nur mit 200 Artilleriegeschützen und 75 Maschinengewehren ausgerüstet, also im Hinblick auf die notwendige Feuerkraft unterlegen. Zunächst sorgte der Vormarsch auf breiter Front bei den russischen Befehlshabern für Panik und die Evakuierung der Region wurde diskutiert, allerdings verlangsamte sich der osmanische Vormarsch recht schnell, denn der Mangel an Proviant und Winterkleidung machte sich zusehends bemerkbar. Ein am 25. Dezember einsetzender Schneesturm, der Temperaturen bis $-26^{\circ} \mathrm{C}$ mit sich brachte, sorgte bei der Dritten Armee für zahlreiche Verluste aufgrund von Erfrierungen. Als man vier Tage später Sarıkamış erreichte, waren die osmanischen Soldaten bereits an der Grenze ihrer Belastbarkeit angelangt und hatten den russischen Kontingenten nur wenig entgegenzusetzen. Zwei Frontalangriffe des 9. und 11. Korps wurden zurückgeschlagen, sodass Enver Paschas Armee hohe Verluste erlitt. Mit dem Ende der osmanischen Vorwärtsbewegung, also dem Versacken des Angriffs, gingen die russischen Verbände ihrerseits dazu über, den Feind einzukreisen, wobei das 9. Korps vollständig aufgerieben wurde. Am 4. Januar 1915 mussten sich 
die osmanischen Verbände schließlich nach Erzurum zurückziehen, um einer völligen Niederlage zu entgehen.

Mit dem Beginn des Völkermordes an den Armeniern ${ }^{10}$ im April 1915 sowie den osmanischen Bemühungen, die Gallipoli-Halbinsel gegen die alliierte Invasion zu verteidigen verlagerte sich das militärische Augenmerk Enver Paschas für den Großteil des Jahres, bis schließlich, durch die erfolgreiche Abwehr der Landungsoperationen erneut Truppenkontingente zur Verfügung standen, um die ambitionierten Pläne auf dem Kaukasus weiterzuverfolgen.

Während Mustafa Kemal eine Weiterführung von Operationen im Kaukasus ablehnte und, ebenso wie die deutschen Berater, eher dafür plädierte, die Truppen defensiv zu verwenden, setzte sich Enver Pascha mit seinen Wünschen durch. Anstatt die Truppen in Konstantinopel und Anatolien zu konzentrieren, wurden sie an unterschiedlichen Frontabschnitten zum Einsatz gebracht, eine Entscheidung die ebenfalls die Logistik für die Versorgung der kämpfenden Truppenkontingente erschwerte. Im Dezember 1915 hatte jedoch ein russisches Expeditionsheer unter dem Kommando von Generalmajor Nikolai Baratow (1865-1932) eine Offensive begonnen und im folgenden Verlauf bis März 1916 unter anderem die Städte Kangavar und Isfahan eingenommen. Zudem näherten sich seine Truppen gefährlich nah der Grenze zu Mesopotamien, sodass auch diese Region des Osmanischen Reiches von dem russischen Vorstoß bedroht wurde.

Der Oberbefehlshaber der zaristischen Armee im Kaukasus, Generalleutnant Nikolai Judenitsch (1862-1933), bereitete derweil eine Winteroffensive für Januar 1916 vor. Sein Plan ging auf, so dass die russischen Truppen den osmanischen Gegner in der Schlacht bei Köprüköy überraschen konnten und das 11. Korps den Rückzug antreten musste. Wie schon 1914 hatte die Dritte Armee eine bittere Niederlage erlitten, zudem verfolgte das russische Heer den osmanischen Gegner im Januar 1916 bis zur Festung Erzurum. Der Fall Erzurums am 16. Februar 1916 stellte einen wichtigen Triumph für Judenitsch und die russische Armee dar, ebenso wie es ein Desaster für Enver Pascha und das Osmanische Reich bedeutete. Ostanatolien stand damit schutzlos einem möglichen Vormarsch des Feindes gegenüber und der Schaden, der dadurch für die gesamte Militärmaschinerie der Osmanen entstand, war immens. Abgesehen von hohen Verlusten hatte das militärische Engagement nur Niederlagen gebracht, die die Erfolge andernorts, etwa bei Gallipoli, wieder relativierten.

Darüber hinaus waren die Möglichkeiten weiterer militärischer Operationen aufgrund der Ereignisse eher beschränkt. Infolgedessen sind die Niederlagen in

10 Auf den Völkermord an den Armeniern kann hier nicht ausführlich eingegangen werden. Vgl. dazu: Gerlach, Gesellschaften, S. 124-161. 
den Kampagnen gegen die britischen Verbände in Palästina und Mesopotamien als direkte Folgen der desaströsen Erfahrungen im Kaukasus zu verstehen. Seit dem Sommer 1916 existierte die Dritte Armee de facto nicht mehr. Weitere Operationen im Kaukasus, die im Prinzip nur noch als Schadensbegrenzung dienen sollten, dezimierten zudem die Truppenzahl der Zweiten Armee im Winter 1916/17. Von 800.000 Mann Kampfstärke im Jahr 1915 schrumpfte das osmanische Heer auf 400.000 Mann im März 1917. Die Gallipoli-Kampagne hatte zwar ebenfalls hohe Verluste zu verzeichnen, jedoch lieferte diese ein völlig anderes Ergebnis als das der Kaukasusoperationen, deren desaströse Bilanz in keiner Weise zu kaschieren war.

Ein Vergleich der beiden Operationen zeigt ebenso, dass die Qualität der Offiziere, die Ausbildung, Ausrüstung und Vorbereitung der Truppen, Wissen um die Beschaffenheit des Terrains und damit verbundener Faktoren, wie etwa des Wetters den Unterschied zwischen Erfolg und Katastrophe bedeuten konnten. Während Liman von Sanders die wohl besten Truppen des Osmanischen Reiches befehligte, eiferte Enver Pascha deutschen Glanzleistungen nach, ohne sein Heer entsprechend vorbereitet zu haben. Dahingehend beging er die gleichen Fehler wie seine britischen Kollegen. Aus deutscher Sicht heißt das, dass der Einfluss der Militär-Mission bei Gallipoli am spürbarsten war und durchaus aktiv zu den wenigen Erfolgen des Osmanischen Reiches während des Ersten Weltkrieges beigetragen hatte. Aufgrund dieses Erfolges hatten sich die britischen Kriegsplaner anderen Schauplätzen zuwenden müssen. Zum einen wurde im Oktober 1915 ein britisch-französisches Expeditionsheer nach Saloniki entsandt, ohne jedoch, zumindest vor 1918, wirklich effektiv in das Kriegsgeschehen eingreifen zu können. Das Hauptaugenmerk richtete sich vielmehr auf den Mittleren Osten.

\section{2 Ägypten und Palästina}

Das britische Interesse im Mittleren Osten richtete sich nach dem Misserfolg von Gallipoli auf Ägypten und Palästina, die aus geostrategischer Sicht besonders wichtig waren, um die Kontrolle des Suezkanals und damit des Seeweges nach Indien nicht zu verlieren. $\mathrm{Zu}$ Beginn des Krieges wurden zunächst kleinere indische Kontingente nach Ägypten beordert, da zu diesem Zeitpunkt im War Council noch davon ausgegangen wurde, dass eine Bedrohung des nordafrikanischen Territoriums außer Frage stand. Außerdem hätte die Gallipoli-Kampagne das Osmanische Reich, so die Annahme, schon bald zum Ausscheiden aus dem Krieg gezwungen. Als das nicht geschah, wurden die von der Gallipoli-Halbinsel zurückkehrenden Einheiten, also das Mediterranean Expeditionary Force zur Egyptian Expeditionary Force (EEF) umstrukturiert, bevor schließlich nach Nor- 
den gegen Palästina vorgestoßen werden sollte. Im Gegensatz zu Gallipoli wurde im Zuge der Palästina-Kampagne vorsichtig agiert, besonders da der Einsatz von bis zum Kriegsende immerhin etwa 450.000 Soldaten der Logistik einiges abverlangte.

Eine militärische Bedrohung Ägyptens und des Suezkanals bestand indessen lediglich bis zum 3. Februar 1915. Etwa 20.000 osmanischen Soldaten, unterstützt von einigen wenigen Artilleriegeschützen und deren Mannschaften, war es gelungen, die Wüste Sinai zu durchqueren und die wichtige Verbindung nach Britisch-Indien kurzzeitig zu gefährden. Angeführt wurde das Kontingent von General Friedrich Kreß von Kressenstein (1870 - 1948), dem das Kommando über die 10. und 25. Infanteriedivision aus Cemal Paschas (1872-1922) Vierter Armee in Syrien übertragen worden war. Der Plan bestand darin, den Suezkanal zu blockieren und den parallel verlaufenden Süßwasserkanal zu zerstören. Die indischen Verteidiger waren aber in der Lage, den osmanischen Angriff abzuwehren und dem Gegner schwere Verluste beizubringen. Zuvor konnten jedoch zwei Kompanien den Kanal überqueren und einen deutsch-osmanischen Brückenkopf errichten und zeigen, dass die Einnahme des Suez-Kanals durch feindliche Truppen zumindest im Bereich des Möglichen war.

Später mussten sich die osmanischen Kontingente nach Palästina zurückziehen, da die Verluste zu schwer wogen und die logistischen Voraussetzungen für eine längerfristige Fortsetzung des Angriffs gegen die britischen Stellungen und deren indische Verteidiger nicht gegeben waren. Nachdem die Gefahr eines Verlustes der Kontrolle über den Suez-Kanal damit scheinbar bereits Anfang 1915 gebannt worden war, diente Ägypten im weiteren Verlauf des Frühjahrs lediglich als Stützpunkt für die auf die Gallipoli-Halbinsel beorderten britischen und australischen Soldaten. Im April sollte General Kreß von Kressenstein noch einmal versuchen, die Briten zu überraschen und den Suezkanal einzunehmen. Der Angriff am 23. April 1915 scheiterte erneut und wurde nur unter schweren Verlusten der britischen Truppen abgewehrt, sodass, nachdem die Landungstruppen für Gallipoli Ägypten einige Tage später verlassen hatten, darüber diskutiert werden musste, wie man ähnliche Vorstöße aus Palästina in Zukunft vermeiden könne.

Unabhängig davon wurde von dem deutsch-osmanischen Expeditionskorps aus der Wüste Sinai heraus am 4. August 1916 noch ein letzter Versuch unternommen, den britischen Gegner bei Romani zu überraschen, erneut ohne Erfolg. ${ }^{11}$ Von den 18.000 beteiligten Soldaten auf osmanischer Seite wurden 4.000 Mann

11 Carl Mühlmann, Kriegswerk Bd. XI, Türkei, Der Krieg der Türkei, Potsdam, Oktober 1936, BArch MArch RH61/1827. 
gefangen genommen, nachdem das Unternehmen als solches bereits 9.000 Tote und Verwundete gekostet hatte. Die australischen, neuseeländischen und britischen Verteidiger hatten hier einen wichtigen Sieg errungen. Es konnte ein für allemal verhindert werden, dass das osmanische Heer auf diesem Kriegsschauplatz den strategisch wichtigen Raum für sich beanspruchen konnte. Das deutschosmanische Expeditionskorps, oder was von diesem noch übrig war, musste sich nach Norden zurückziehen. Der Sieg bei Romani war mit Blick auf die Dimension sicherlich keine Kompensation für Gallipoli, aber es deutete sich eine Wende im alliierten Kriegsglück im Mittleren Osten an, in erster Linie mit einer besseren Planung und Vorbereitung auf Seiten der alliierten Befehlshaber zusammenhing. Der britische Sieg markierte darüber hinaus die Transformation der Sinai-Kampagne von einer defensiven zu einer offensiven Ausrichtung zukünftiger Operationen. Es ging nun nicht mehr um die Verteidigung des Suezkanals, sondern vielmehr um die Einnahme osmanischen Territoriums.

Im März 1917 wurden vom EEF die ersten Angriffe in Palästina durchgeführt, zum Beispiel beim Vorstoß auf Gaza am 26. März. Dabei handelte es sich nicht um ein nur lokales Vorgehen. Die Offensivpläne der britischen Kontingente in Palästina liefen parallel zu den flächendeckenden Angriffen der Alliierten an allen Frontverläufen, die Ende Februar 1917 bei einer anglo-französischen Konferenz in Calais besprochen worden waren. Nachdem die britischen Streitkräfte im April 1916 bei Kut al-Amara in Mesopotamien eine erhebliche Niederlage zu beklagen hatten, waren die politischen Entscheider in London, allen voran Premierminister Lloyd George nicht gewillt, weiterhin halbherzig in dieser wichtigen Region des britischen Empire zu agieren. Zudem hoffte man darauf, dass eine erfolgreiche Operation gegen die Osmanen in Palästina auch Aufstände der arabischen Stämme motivieren würde, die dann osmanische Truppen im Süden binden würden, was den Russen im Norden und den Briten in Mesopotamien Entlastung verschaffen sollte.

Der Angriff auf Gaza am 26. März 1917 war jedoch lediglich der erste von letztlich drei Versuchen, den Besatzern die Stadt zu entringen. Auch wenn der anfängliche Kavallerieangriff erfolgversprechend schien, konnten die britischen Kontingente von den osmanischen Verteidigern zurückgeschlagen werden. Der zweite Angriff vom 17. bis 19. April 1917 scheiterte ebenfalls, und das, obwohl dabei zum ersten Mal Giftgas im Mittleren Osten zum Einsatz kam. Die inzwischen verstärkten osmanischen Truppen konnten den britischen Angriff zurückschlagen, was sich schlecht auf die Moral der Soldaten des EEF auswirken musste. Die zweite britische Niederlage führte schließlich dazu, dass der Kommandeur des EEF, General Archibald Murray (1860-1945), abberufen und am 11. Juni 1917 durch General Edmund Allenby (1861-1936) ersetzt wurde. Für letzteren war die Ernennung keine Beförderung, sondern kam eher einer Bestrafung durch Ver- 
setzung gleich, nachdem Allenby in der Schlacht bei Arras im April 1917 keinen britischen Durchbruch an der Westfront erreicht hatte.

Den Umständen der Versetzung ungeachtet hatte Allenby die Unterstützung Lloyd Georges, der ihn ermahnt hatte, dass das Britische Empire dringend einen Sieg benötige, um die Moral der Truppen wieder zu verbessern. Gaza war das Ziel für Allenby und eine Niederlage war aus politischer Sicht unbedingt zu vermeiden. Daher wurden mehr Ressourcen zur Verstärkung des Eisenbahnnetzes in Richtung Gaza gewährt, um die Logistik und damit die Versorgung der britischen Angreifer zu verbessern. Zudem wurde im Kriegskabinett in London an einem neuen Angriffsplan gearbeitet, der vorsah, die osmanischen Truppen erst von der Wasserversorgung bei Be'er Scheva (heute im südlichen Israel) abzuschneiden, um dann gegen einen geschwächten Feind in Gaza vorzurücken. Zwischen dem 31. Oktober und 7. November 1917 kam es schließlich zur dritten Schlacht um Gaza, die mit dem schwersten Artilleriefeuer des Ersten Weltkrieges außerhalb Europas begann. Am Ende zeigte die sorgfältige Vorbereitung in Verbindung mit ausreichend Nachschub und Munition Wirkung: Dem seit Mitte 1917 Oberkommandierenden der osmanischen Armee, General Erich von Falkenhayn (1861-1922) blieb nichts Anderes übrig, als die verbliebenen Kräfte der Siebten und Achten Armee nach Jerusalem zurückzuziehen.

Die osmanischen Truppen versuchten in mehreren Anläufen im Laufe des Novembers 1917, den britischen Vormarsch in Richtung Jerusalem zu stoppen, die Stadt fiel allerdings am 9. Dezember. Der Einmarsch Allenbys und seiner Soldaten wurde in den britischen Zeitungen zu einem der größten Erfolge Großbritanniens in Palästina, ja sogar zum Sieg in einem modernen Kreuzzug stilisiert. Ein osmanischer Versuch, die Stadt am 27. Dezember zurückzuerobern, scheiterte, obwohl das EEF mehr als 1.000 Mann Verluste zu verzeichnen hatte. Selbst wenn die osmanische Armee danach noch über Kontingente in Palästina verfügte und es bis Februar 1918 zu kleineren Scharmützeln im Raum um Jerusalem kam, war der Fall von Jerusalem ein deutlicher Beleg für die Schwäche des osmanischen Gegners. Schon am 13. Dezember 1917 hatte Liman von Sanders die Folgen der Niederlagen im Kaukasus und Palästina zusammengefasst: „Durch eine Anzahl verfehlter Massnahmen ist das tuerkische Heer zu einem auffallenden Tiefstand an Kopfzahl der fechtenden Truppen, sowie zu einem nicht zu unterschaetzenden Rueckgang in der Kriegstuechtigkeit, gelangt.“12

Die militärischen Aktivitäten in Palästina sollten jedoch im September 1918 noch einmal Fahrt aufnehmen und in der Schlacht bei Megiddo zu einer letzten

12 Liman von Sanders, Der heutige Zustand der tuerkischen Armee, Panderma, 13. Dezember 1917, BArch MArch N247/40, S. 1. 
direkten Konfrontation von Einheiten des Osmanischen Reiches und den Kontingenten des EEF führen. In einem mobilen Gefecht nutzte Allenby seine Infanterie und Kavallerie zur Attacke, die von Flugzeugen und Transportfahrzeugen unterstützt wurden, um die im Rückzug befindliche osmanische Armee von einem möglichen Fluchtweg abzuschneiden. So konnten mehr als 75.000 Soldaten der osmanischen Armee während der britischen Offensive gefangen genommen werden, bis diese am 26. Oktober 1918 bei Aleppo schließlich stoppte. Allenby konnte bei seinen Operationen durchaus sehr schnell vorrücken, wobei hier angemerkt werden muss, dass osmanischer Widerstand oft völlig ausblieb, da die demoralisierten Truppen nicht gewillt waren, den Raumgewinn des Gegners um jeden Preis zu verhindern. Mit dem Waffenstillstand von Mudros am 30. Oktober 1918 endete die Kampagne in Palästina, ebenso wie die Kriegsteilnahme des Osmanischen Reiches. Die Grundsteine für die bis heute andauernden politischen Probleme der Region wurden im Anschluss an die militärischen Operationen gelegt und sollen hier nicht eingehender diskutiert werden. Entscheidend ist mit Blick auf die Gallipoli-Kampagne, dass die britischen Kontingente in Palästina dort mit ähnlichen Problemen zu kämpfen hatten. Erst die Einsicht, dass mehr zur Unterstützung der Truppe getan, also eine bessere Logistik vorbereitet und ein Mehr an Nachschub und Munition garantiert werden musste, sorgte schlussendlich dafür, dass die britischen Operationen in Palästina erfolgreicher waren als bei Gallipoli.

\subsection{Mesopotamien}

Das Zweistromland bildete ebenfalls einen Konfliktraum zwischen 1914 und 1918, in dem die Interessen des Osmanischen Reiches mit denen des britischen Empire kollidierten. Der Erste Weltkrieg sollte dort eine bis heute geopolitisch bedeutsame Rolle spielen, da im Zuge der Ereignisse 1921 der Irak in seinen heutigen Grenzen entstand. ${ }^{13}$ Zunächst oblag die militärische Verantwortung für diesen Kriegsschauplatz der Regierung von Britisch-Indien und deren Vertretern im India-Office in London. Die ersten Operationen in Mesopotamien wurden von indischen Truppen bestritten. Es waren die Gegensätze zwischen den britischen Interessen im Mittleren Osten und der tatsächlichen Handhabe durch das IndiaOffice, die zu Reibungen und schließlich zu Schwierigkeiten in der Durchführung der Kampagne in diesem Gebiet führten. Gegensätzliche Meinungen in London,

13 Ausführlich behandelt Krethlow (Bagdad) die Kampfhandlungen im späteren Irak. 
Kairo und Neu-Delhi erschwerten einen einheitlichen Kurs und damit konkrete Planungen sowie den Erfolg der militärischen Operationen in Mesopotamien.

Die osmanischen Truppen in Basra hatten sich zunächst eher defensiv verhalten als die indischen Militärbehörden nach dem Kriegseintritt der Pforte die 18. Infanteriebrigade zur Verstärkung der in Mesoptamien stationierten 16. Brigade abkommandiert hatten. General Arthur Barrett (1857-1926), der für dieses Operationsgebiet verantwortlich war, erhielt Mitte November 1914 den Befehl aus London, auf Basra vorzurücken. Am 17. November kam es zu einem ersten Aufeinandertreffen der beiden Armeen bei Salih, in dem sich die britischen Einheiten durchsetzen und im Anschluss daran schließlich Basra einnehmen konnten. Die schweren Verluste auf beiden Seiten beim ersten Gefecht bei Salih - 500 Mann auf britisch-indischer, 1000 auf osmanischer Seite waren verwundet oder getötet worden - zeigten bereits, dass der Krieg in Mesopotamien kein einfaches Unterfangen werden würde.

Barrett rückte schließlich weiter vor und überdehnte damit nicht nur das Transportnetzwerk, das ihm in der Region zur Verfügung stand, sondern dünnte gleichzeitig die Mannstärken seiner Truppen an den unterschiedlichen Frontabschnitten aus. Zudem war die Koordination der einzelnen Truppenbewegungen immer noch auf die militärischen Planer in London und Neu-Delhi verteilt, sodass keine einheitliche Strategie entwickelt werden konnte. Die bis 1916 andauernde Vorwärtsbewegung Barretts sollte sich deshalb schlussendlich nicht auszahlen. Während die indische Seite auf die Ausweitung des Operationsgebietes drängte, schon um einen ausreichenden Puffer zwischen sich und dem Osmanischen Reich zu wissen, war das allgemeine Interesse an einer solchen Intensivierung der Kampagne eher verhalten. Denn zu Beginn des Jahres 1916 wog das Desaster von Gallipoli noch zu schwer, um schon wieder ein neues Operationsgebiet im Mittleren Osten zu favorisieren. Ungeachtet dessen genehmigten die Entscheidungsträger in Neu-Delhi und im Londoner India-Office, deren geostrategisches Interesse an der Region größer war als das der Regierung, weitere Operationen Barretts in Mesopotamien, zumal mit einer möglichen Einnahme Bagdads ein prestigeträchtiger Erfolg in Aussicht stand.

Ein erster Versuch der britisch-indischen Truppen, am 4. Dezember 1914 AlQurna einzunehmen, scheiterte an der schlechten Vorbereitung, die sich in einem Mangel an Transporttieren sowie einer unzureichenden Wasserversorgung der Soldaten widerspiegelte. Man musste sich nach Basra zurückziehen, sodass die Stadt erst in einem zweiten Anlauf am 9. Dezember eingenommen werden konnte. Ab diesem Zeitpunkt waren nur noch die Entscheidungsträger in Indien sowie die Offiziere vor Ort verantwortlich, da sich das India Office in London aus den Planungen weiterer Operationen heraushielt. Dieser Umstand sorgte dafür, dass sich die Zielsetzung der Mespotamien-Kampagne entscheidend veränderte. Aus einem 
defensiv ausgerichteten Militäreinsatz zur Verteidigung des Persischen Golfs wurde eine breit angelegte offensive militärische Unternehmung, die in erster Linie von der Euphorie der Planenden - ganz ähnlich wie im Falle Gallipolis getragen wurde. Im Januar 1915 wurde eine dritte Infanteriebrigade aus Indien abkommandiert. Im Laufe des Jahres überdehnte Barrett alle Kapazitäten seines Transport- und Nachschubnetzwerkes. Unzusammenhängende Operationen, wie etwa gegen Amara am Tigris und Nassirija am Euphrat wurden zwar ausgeführt und im April 1915 konnte eine osmanische Gegenoffensive erfolgreich abgewehrt werden, so dass alles den Entscheidungsträgern in Britisch-Indien Recht zu geben schien. Aber mit dem Eintreffen osmanischer Verstärkungen aus Bagdad sollte sich das Blatt schließlich zu Ungunsten des Empire wenden.

In Anbetracht der festgefahrenen Situation auf der Gallipoli-Halbinsel waren die Erfolgsmeldungen aus Mesopotamien zunächst wie Balsam für die geplagten Mitglieder des War Council, die jedoch auch hier nicht erkannten, dass die Realität vor Ort ganz anders war als das, was man sich mit viel Zuversicht bereits als Vorbereitung der britischen Einnahme Bagdads, natürlich im Handstreich, ausmalte. Der im September 1915 beginnende Vormarsch auf Kut al-Amara war nicht ausreichend vorbereitet worden. So gab es kaum motorisierte Fahrzeuge und die militärische Führung um Barrett verließ sich auf den Gebrauch von Transporttieren, wie in der indischen Armee üblich. Das erschwerte ebenfalls den Transport der notwendigen Artillerie und des Nachschubs, sodass sich die britisch-indischen Truppen im Februar 1916, nachdem es zu mehreren Schlachten mit der osmanischen Armee nördlich von Kut al-Amra gekommen war, wieder dorthin zurückziehen mussten. General Charles Townshend (1861-1924), der den britischen Vormarsch kommandiert hatte, entschied sich schließlich dazu, in Kut alAmra auszuharren, bis Verstärkungen aus Basra einträfen. Dazu ließ er die Stadt befestigen und zu einem militärischen Fort umbauen.

Hier offenbarten sich jedoch die Probleme mit dem Nachschub ganz deutlich. Am 29. April 1916 musste Townshend nach gut vier Monaten des Ausharrens kapitulieren. Die erneute Schmach der britischen Armee war allen Beteiligten und auch allen Beobachtern bewusst. Erneut hatte das Empire versagt. Erneut waren die osmanischen Soldaten diejenigen, die die Oberhand behielten. Das Prestige Großbritanniens bröckelte, nicht nur an der Westfront, aber vor allem in den Kolonien, in denen die militärischen Operationen gegen das Osmanische Reich genau verfolgt wurden.

Erneut bedurfte es eines Schockmoments, bevor Planer und Entscheidungsträger erkannten, dass auch der Krieg gegen das Osmanische Reich nicht ohne entsprechenden Einsatz an Material zu gewinnen war. Eine Reorganisation des britisch-indischen Heeres auf dem Kriegsschauplatz Mesopotamien setzte ein und tatsächlich war es Generalleutnant Stanley Maude (1864-1917), der elf Monate 
nach Kut al-Amara am 11. März 1917 in Bagdad einmarschierte. Zuvor waren die Truppen in Mesopotamien zur Mesopotamian Expeditionary Force (MEF) umstrukturiert und mit besserer Ausrüstung versehen worden. Im War Office hatte General Charles Monro die Planungen übernommen und mit Maude einen fähigen und erfahrenen Offizier zum Kommandeur des MEF berufen. Maude, der seit seinem Einsatz auf der Gallipoli-Halbinsel unter dem Spitznamen „Systematic Joe“ bekannt war, schien genau der richtige Mann für die ihm zugedachte Aufgabe. Nachdem er zunächst den Ausbau der Hafenanlagen in Basra im Herbst 1916 überwacht hatte, so dass wesentlich mehr Schiffe mit mehr Nachschub gleichzeitig entladen werden konnten, nahm Maude die Operationen mit dem Ziel Bagdad Mitte Dezember 1916, mit einem Angriff der osmanischen Positionen bei Hai, wieder auf. Trotz einer Unterbrechung der Operation wegen schweren Regens konnte die Stadt am 19. Januar 1917 eingenommen werden. Das MEF operierte im Zuge der Gefechte exzellent, wobei zunächst schwere Artillerie zum Beschuss des Gegners eingesetzt wurde, bevor die Infanterie unter dem Schutz von Maschinengewehrfeuer gegen feindliche Stellungen vorrückte.

Am 4. März 1917 wurde vom Generalstab in London schließlich grünes Licht für den Vormarsch auf Bagdad gegeben. Die Einnahme der Stadt war nicht nur ein militärischer, sondern vor allem auch ein politischer Triumph. Es war der erste große Erfolg des Empire im Mittleren Osten. Maude erklärte sich und seine Soldaten vor der Bevölkerung Bagdads zu deren Befreiern. Trotz der Ausschlachtung dieses wichtigen Sieges der britischen Armee, dauerte der Krieg in Mesopotamien weiter an. Im Winter 1917 und Frühjahr 1918 kam es erneut zu Kämpfen mit der osmanischen Armee, die aber kaum einen Einfluss auf den weiteren Verlauf des Krieges nahmen. Im Gegensatz zu Gallipoli gelang es den britischen Offizieren und Soldaten in Palästina und in Mesopotamien, das Kriegsglück zu wenden und sich zu rehabilitieren. Im Fall von Gallipoli blieb diese Möglichkeit verwehrt. 